\title{
Palynological Study of the Geranium L. Species in Iraq
}

\author{
Sirwan Hassan Salih \\ Biology Department, College of Education, University of Garmian
}

\begin{abstract}
The pollen grains morphology of ten species of Geranium were investigated, two types of pollen surface configuration were found reticulate/gemmate and striate reticulate as well as the results showed two groups of species in pollen sizes the first with less than $100 \mu \mathrm{m}$ of diameter and the other with more the $100 \mu \mathrm{m}$ all illustrated by figures and tables. The average value of pollen polar axis ranged between $60-94 \mu \mathrm{m}$ and equatorial diameter ranged between $58 \mu \mathrm{m}-85 \mu \mathrm{m}$.
\end{abstract}

\section{1- Introduction:}

The application of pollen morphology in recognition and interpretation of relationships of plants at various hierarchial level in plant taxonomy and production of pollen keys has been reported in many angiosperm families (Erdtman 1966, Mulder 2003, Huysmans and et al. 2003, Al-Quran.2004), moreover the advent of scanning techniques has greatly enabled the study of pollen morphology with great precision, thereby making their application much more efficient and dependable. The pollen morphology of Geraniaceae or some of its representative has been studied by many researchers such as Bortenschlager (1967), Olth mann (1967), Verhoeven and Venter (1987), Stafford and Blackmore (1991) and Stafford and Gibby (1992). Recently Aedo, et al. (2007) who have used the palynological data in combination with morphological data in their taxonomic revision of Genus Geranium. Based on previous studies and investigations the pollen morphology of studied species in Iraq had not been described or studied previously.

The main objective of the present study was to investigate and describe the pollen morphology of the Geranium species those distributed in Kurd istan region-Iraq and to show how far the pollen mo rphological variations could be used to make distinction between the studied taxa.

2- Materials and Methods:

This study included 10 species of genus Geranium. Pollen samples of examined species was obtained from filed collections as well as from herbarium specimens (table 1). Pollen grains were prepared for light microscopy (LM) by using the standard method described by Erdtman (1960). Pollens mounted on glycerin jelly onto glass slides and stained with safranine finally observations were made with a Seizz mic roscope under E40 and E100 with 10x eyepiece. Pollen diameter, polar axis (P) and equatorial view (E) were measured on 20-25 pollens of each species and P/E was calculated and all of which were photographed by modern dig ital camera (SONY, 3x, 13.6 Mega pixels). The terminology was used accordance to Erdtman (1969), Walker and Doyle (1976) and Hesse and et al (2009)

3-Results and Discussion:

The results showed that the pollen grains were usually monad, isopolar, radially symmetrical, the mean value of polar axis $(\mathrm{P})$ ranged between $60 \mu \mathrm{m}$ in G. rotundifolium and $94 \mu \mathrm{m}$ in G. stepporum, while the equatorial diameter (E) ranged between $58 \mu \mathrm{m}$ in G. molle and $85 \mu \mathrm{m}$ in G. dissectum (figure 1 and 2), then the size of grains in both polar and equatorial view of all examined species is approximately similar, visually the distances among pores are mostly equal. Pollen outline are circular to slightly triangular or semi ovate with convex sides in polar view shows circular to semi ovate in (G. pusillum, G. divaricatum. 
G. stepporum and G. tuberosum) to elliptic in equatorial view in the others (table 2, figures 3). According to the pollen sizes, the studied species can divide into two groups, the first group included species with medium and large size pollen grains $(41-98 \mu \mathrm{m})$ as in G. divaricatum, G. kurdicum, G. molle, G. purpureum, G. pusillum and G. rotundifolium and the second group involved species with large pollen

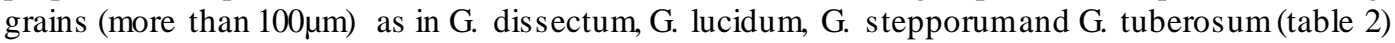

Apertures (pores) tricolporate and the distances among them are usually equally organized. Tectum showed two types sculptures, reticulate/gemmate in B. multifida, G. dissectum, G. molle and G. purpureum whereas grains with minute appendages like gemmate and striate reticulate, in remain species the sculpture distinguished by the existence of straight reticulate ornamentation. The results of pollen morphology are ag reed with the results of Erdtman (1971), Perveen (1999) and Shehata (2008). The two types of pollen in terms of surface ornamentations and exine structures recognized, type I: reticulate/gemmate is readily distinguished by the reticulate tectum which is heavily ornamented with bacula and gemmae, this type is characteristic of G. dissectum, G. divaricatum, G. molle and G. purpureum ant the type II: striate reticulate tectum is characterized by a striate reticulate tectum were found in other remain species. Generally the pollen dissimilarities of these species are little, the apertures diameters and distances among them are semi- equal also the colors of pollen grains are ranged from light yellow, yellowish white to dirty white. However the most important features of pollen are the sculptures or ornamentation that could be used for splitting infrageneric taxa into groups and that which need to be studied in detail in future by scanning electron microscope (SEM) to show the variations obviously.

Table (1) Plant specimens used for palynology.

\begin{tabular}{|l|l|c|l|l|}
\hline \multicolumn{1}{|c|}{ species } & $\begin{array}{l}\text { Specimens } \\
\text { number }\end{array}$ & Herbarium & \multicolumn{1}{|c|}{ Localities } & \multicolumn{1}{c|}{$\begin{array}{c}\text { Collection } \\
\text { Date }\end{array}$} \\
\hline & 1308 & SUH & Kalar (Sarqala) & $22-3-2008$ \\
Geranium dissectum & 2712 & SUH & Near Penjwen & $10-5-1978$ \\
& 1058 & SUH & Sakran mt. & $1-5-2008$ \\
& 373 & SUH & Kani Watman & $8-4-2008$ \\
\hline & 0013116 & BUH & & \\
G. divaricatum & 0013119 & BUH & Halgurd mt. & $28-5-1961$ \\
& & & & \\
\hline & 0013121 & BUH & & $7-6-1961$ \\
G. kurdicum & 0013122 & BUH & Halgurd mt. & $7-6-1961$ \\
& 0013117 & BUH & & $15-8-1956$ \\
\hline & 927 & SUH & Bekhal /Rowanduz & $1-5-2008$ \\
G. lucidum & 5352 & SUH & Piramagroon mt & $23-5-1981$ \\
& 233 & SUH & Ahmedawa/ Khurmal & $3-4-2008$ \\
& 1282 & SUH & Sartaky Bamo mt. & $20-3-2008$ \\
\hline \multirow{5}{*}{ G. molle } & 1276 & SUH & Sartaky Bamo mt. & $20-3-2008$ \\
& 5363 & SUH & Piramagroon mt. & $16-1981$ \\
& 1028 & SUH & Gali Akoian/ Erbil & $1-5-2008$ \\
& 2280 & SUH & Qashqoly/ Dukan & $12-4-2008$ \\
\hline \multirow{5}{*}{ G. purpureum } & 0013137 & BUH & Shaqlawa/ Erbil & $11-4-1960$ \\
& 241 & SUH & Ahmedawa/ Khurmal & $3-4-2008$ \\
& 1019 & SUH & Gali Akoian/ Erbil & $1-5-2008$ \\
& 741 & SUH & Bargalu/ Sulaimany & $17-4-2008$ \\
\hline G. stepporum & 996 & SUH & Gali Akoian/ Erbil & $1-5-2008$ \\
& 1039 & SUH & Zerenok/ Rowanduz & $1-5-2008$ \\
& 1047 & SUH & Galala/ Erbil & $1-5-2008$ \\
& 1249 & SUH & Kani Bazi/ Amedy & $2-5-2008$ \\
\hline & 0013145 & BUH & Qopy Qaradagh & $18-6-1959$ \\
& 1112 & SUH & Gali Zanta/ Aqra & $2-5-2008$ \\
& 147 & SUH & Awa Khweri/ Kalar & $31-3-2008$ \\
& 912 & SUH & Piramagroon mt & $24-4-2008$ \\
\hline & 784 & SUH & Haibat Sultan mt. & $17-4-2008$ \\
\hline
\end{tabular}




\begin{tabular}{|l|l|l|l|l|}
\hline & 398 & SUH & Azmer mt. & $9-4-2008$ \\
& 823 & SUH & Mlakawa/ Penjwen & $18-4-2008$ \\
& 1052 & SUH & Sakran mt./ Erbil & $1-5-2008$ \\
\hline & 0029689 & BUH & Near Duhok & $4-5-1976$ \\
G. tuberosum & 0013160 & BUH & Dukan & $2-4-1967$ \\
& 2002 & SUH & Sarser/ Tawela & $22-4-2009$ \\
& 1256 & SUH & Ahmedawa/ Khurmal & $3-4-2008$ \\
\hline
\end{tabular}

Table (2). Dimensions and Morphology of Pollen grains.

\begin{tabular}{|c|c|c|c|c|c|c|}
\hline \multirow{2}{*}{ species } & \multicolumn{2}{|c|}{ Polar axis } & \multicolumn{2}{|c|}{ Equatorial axis } & \multirow{2}{*}{$\begin{array}{c}\text { Exine } \\
\text { element } \\
\text { thickness } \\
(\mu \mathrm{m})\end{array}$} & \multirow{2}{*}{$\begin{array}{c}\text { Surface area } \\
\text { (P. a. length } x \\
\text { E.a.length) } \\
(\mu \mathrm{m})\end{array}$} \\
\hline & $\begin{array}{c}\text { Diameter } \\
(\mu \mathrm{m})\end{array}$ & Shape & $\begin{array}{l}\text { Diameter } \\
(\mu \mathrm{m})\end{array}$ & Shape & & \\
\hline Geranium dissectum & $\begin{array}{c}(68-107) \\
88\end{array}$ & $\begin{array}{l}\text { triangular, } \\
\text { semi ovate }\end{array}$ & $\begin{array}{c}(61-102) \\
85\end{array}$ & circular & $(2-5) 4$ & $88 \times 85=7480$ \\
\hline G. divaricatum & $\begin{array}{c}(61-85) \\
76\end{array}$ & triangular & $(53-71) 60$ & $\begin{array}{l}\text { circular, } \\
\text { elliptic }\end{array}$ & (2-4) 3 & $76 \times 60=4560$ \\
\hline G. kurdicum & $\begin{array}{c}(65-98) \\
83\end{array}$ & Triangular & $(60-91) 80$ & $\begin{array}{l}\text { circular, } \\
\text { elliptic }\end{array}$ & (2-4.5) 3 & $83 \times 80=6640$ \\
\hline G. lucidum & $\begin{array}{c}(72-106) \\
90\end{array}$ & $\begin{array}{l}\text { triangular, } \\
\text { circular }\end{array}$ & (57-97) 79 & $\begin{array}{l}\text { circular, } \\
\text { semi ovate }\end{array}$ & $(2.5-5) 4$ & $90 \times 79=7110$ \\
\hline G. molle & $\begin{array}{c}(54-81) \\
68\end{array}$ & $\begin{array}{l}\text { triangular, } \\
\text { circular }\end{array}$ & $(48-65) 58$ & $\begin{array}{l}\text { circular, } \\
\text { semi ovate }\end{array}$ & (2-4.5) 3 & $68 \times 58=3944$ \\
\hline G. purpureum & $\begin{array}{c}(60-83) \\
75\end{array}$ & $\begin{array}{l}\text { triangular, } \\
\text { circular }\end{array}$ & (41-74) 60 & circular & $(1.5-4) 3$ & $75 \times 60=4500$ \\
\hline G. pusillum & $\begin{array}{c}(55-88) \\
74\end{array}$ & triangular & $(55-82) 69$ & $\begin{array}{l}\text { circular, } \\
\text { semi ovate }\end{array}$ & $\begin{array}{c}(2-4.5) \\
3.5\end{array}$ & $74 \times 69=5110$ \\
\hline G. rotundifolium & $\begin{array}{c}(49-72) \\
60\end{array}$ & $\begin{array}{l}\text { triangular, } \\
\text { circular }\end{array}$ & (50-67) 59 & $\begin{array}{l}\text { circular, } \\
\text { semi ovate }\end{array}$ & (2-5) 3.5 & $60 \times 59=3540$ \\
\hline G. stepporum & $\begin{array}{c}(75-107) \\
94\end{array}$ & triangular & $(67-98) 82$ & $\begin{array}{l}\text { circular, } \\
\text { semi ovate }\end{array}$ & (1.5-4) 3 & $94 \times 82=7708$ \\
\hline G. tuberosum & $\begin{array}{c}(60-106) \\
87\end{array}$ & $\begin{array}{l}\text { triangular, } \\
\text { semi ovate }\end{array}$ & $\begin{array}{c}(59-100) \\
81\end{array}$ & $\begin{array}{l}\text { circular, } \\
\text { elliptic }\end{array}$ & $\begin{array}{c}(2-4.5) \\
3.5\end{array}$ & $87 \times 81=7047$ \\
\hline
\end{tabular}




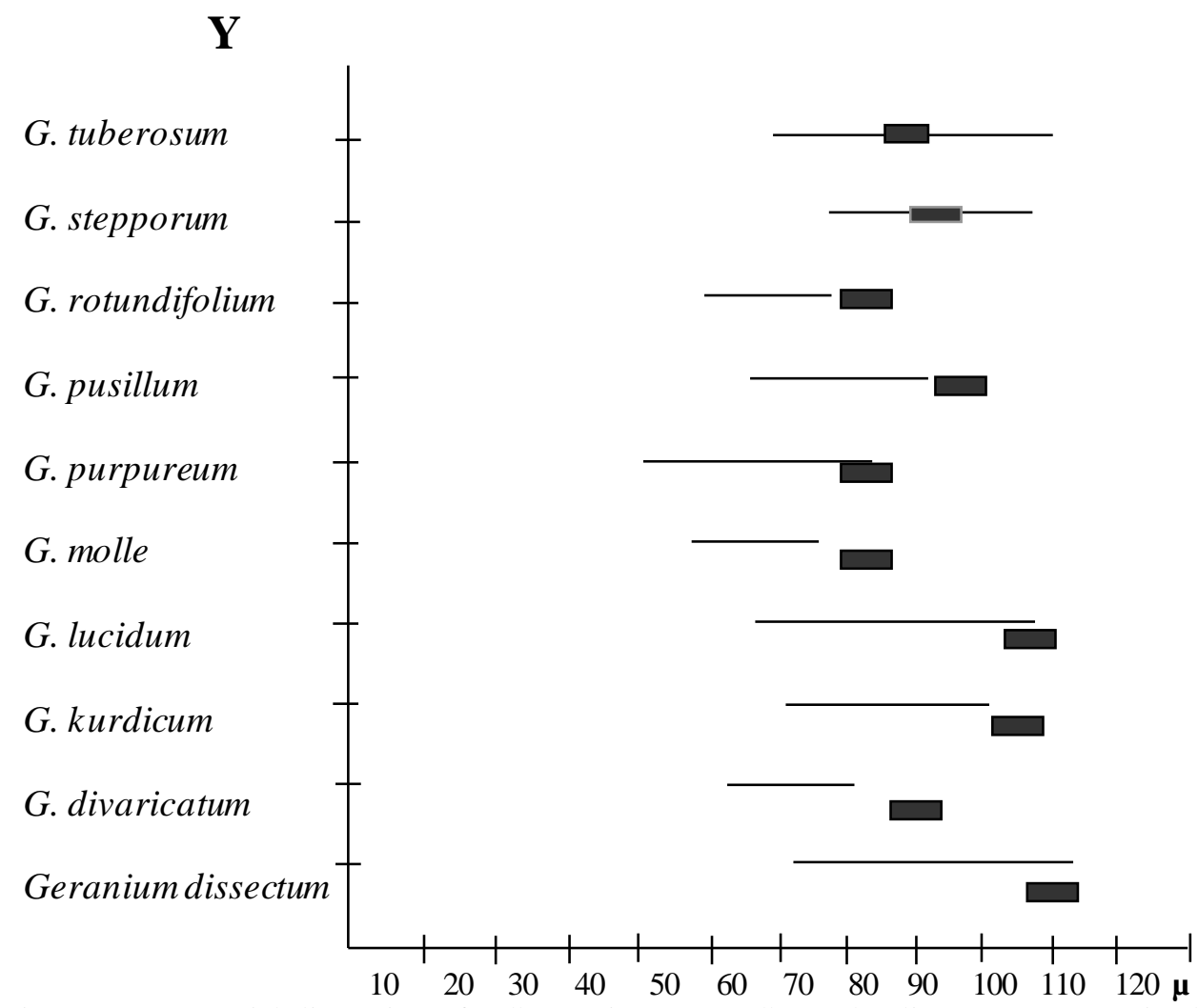

Figure (1) Equatorial dimension of pollen grains $(\mathrm{X}=$ pollen polar diameters $\mathrm{Y}=$ examined species)

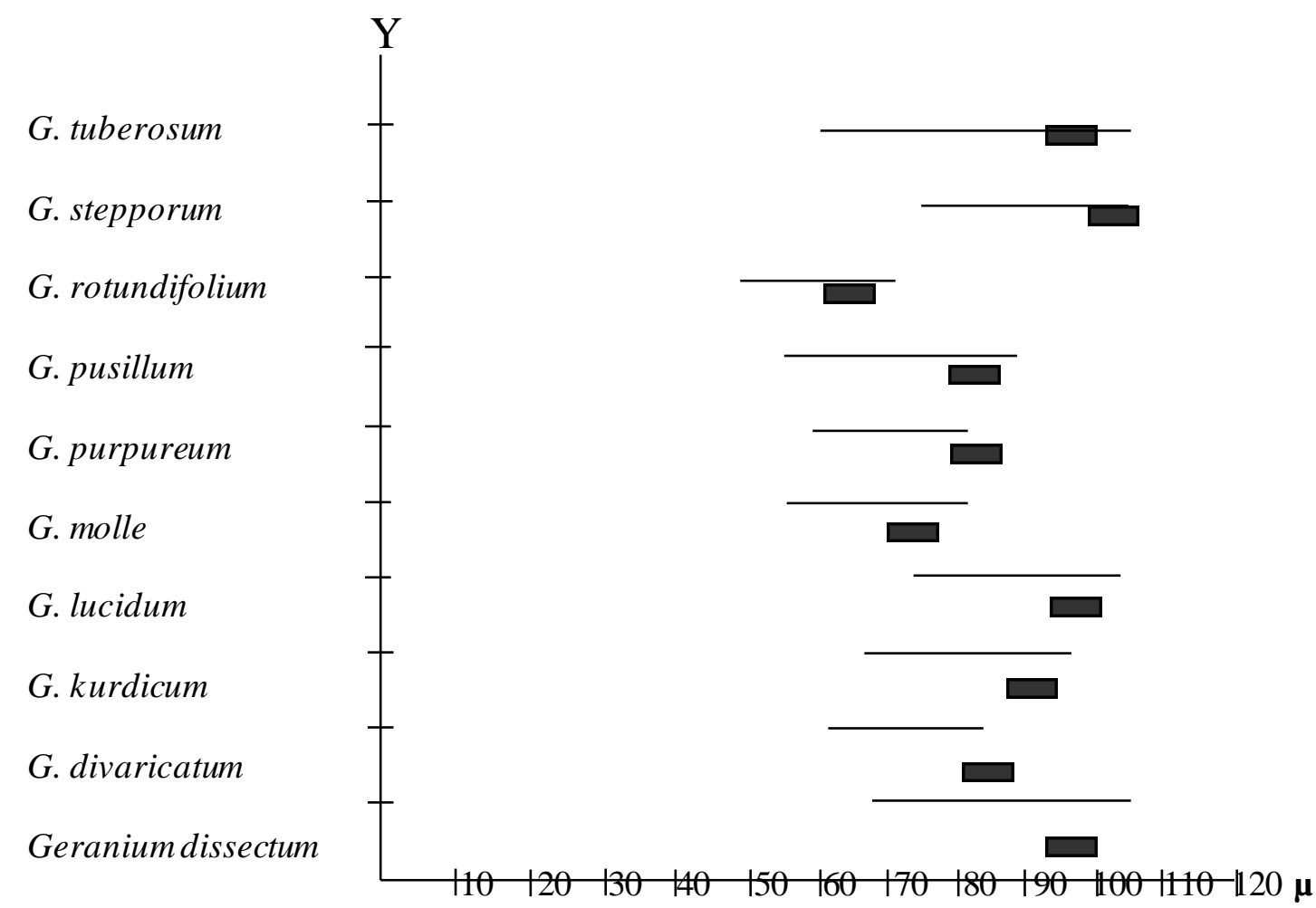

Figure (2) Polar dimensions of pollen grains. ( $\mathrm{X}=$ pollen polar diameters $\mathrm{Y}=$ examined species) 

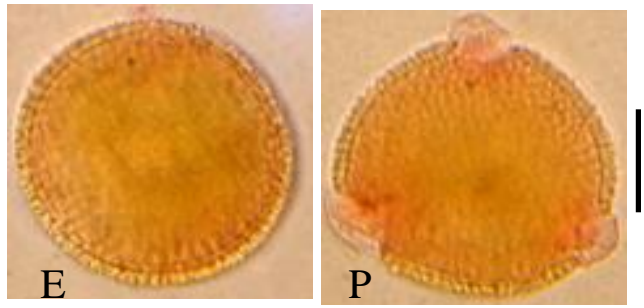

G. divaricatum
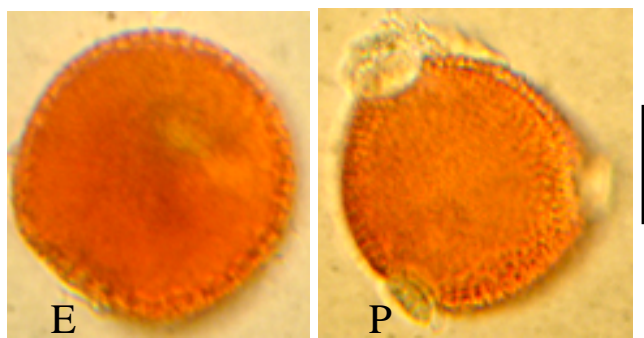

G. molle
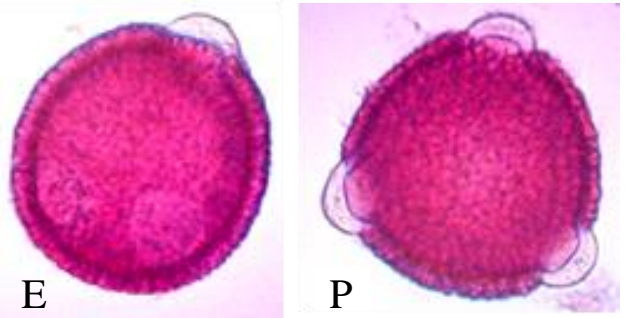

G. rotundifolium

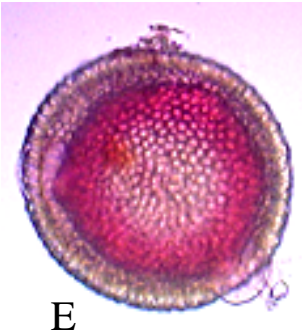

G. purpureum

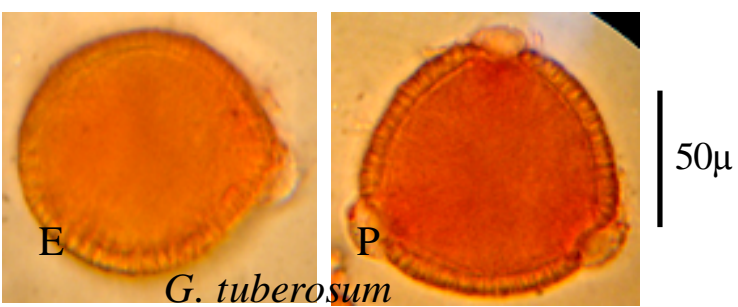

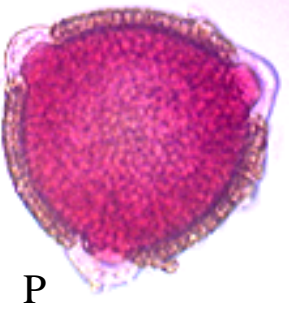

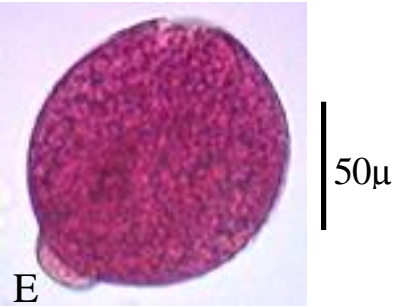

G. dissectum

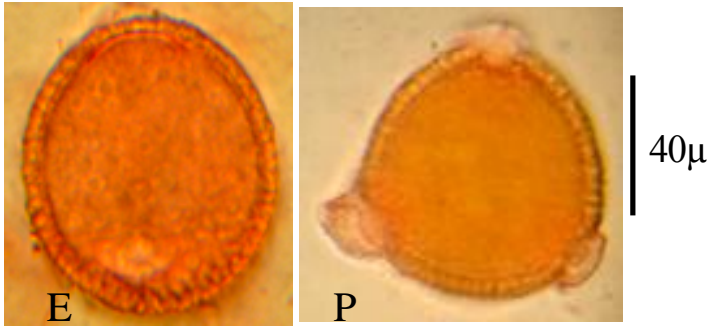

G. pusillum
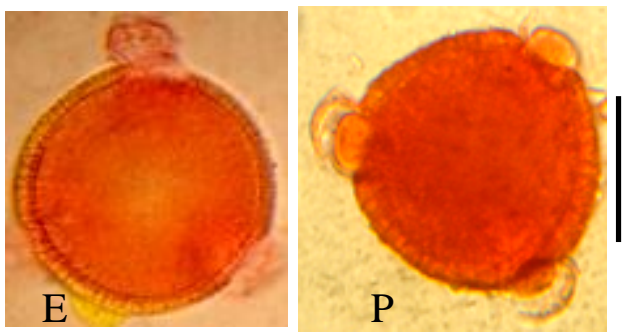

G. lucidum

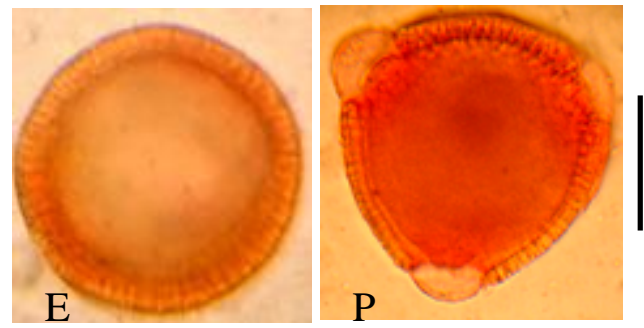

G. stepporum $55 \mu$

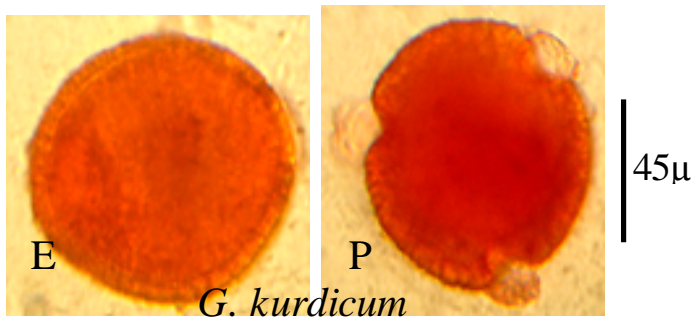

Figure (3) Pollen grain morphology of studied species. (E= Equatorial view, $\mathrm{P}=$ polar view) 


\section{References}

- Aedo, C. et al. 2007. Taxonomic revision of Geranium Subsect. Mediterranean (Geraniaceae). Syst. Bot. 32. pp. 93-128.

- Al-Quran, S. A. 2004. Pollen morphology Plantaginaceae in Jordan. Pak. J. Bio. Sc. pp. 1594-1602.

- Bortenschlager, S. 1967. Vorlaufige Mitteilungen zur Pollen morphologie in der Sequences from der Geraniaceen und inher systimatiche Bedeutumg. Grana play. pp. 400-468.

- Erdtman, G. 1966. Pollen Morphology and Plant Taxonomy. Angiosperm. Hafner publishing Co. New York.

- Erdtman, G. 1969. Handbook of Palynology. pp. 148, 313.

- Erdtman, G. 1971. Pollen morphology and plant taxonomy, angiosperms. New York. pp. 186-187.

- Hesse, M. et al 2009. Pollen Terminology an illustrated handbook. Univ. of Vienna. pp. 4-64.

- Huysmans, S. S. et al 2003. Pollen morphology of NW European representatives confirms morphology of Robieae (Rubiaceae). Rev. Paleobot. Palynol. 127. pp. 219-240.

- Mulder, Ch. 2003. The Northwest European Pollen Flora, 61 Aizoaceae Rev. Paleobot. Palynol. 123. pp. 41-45.

- Olthmann, O. 1967. Pollen morphologich-systematische Unzwrsuchungn innerhalb der Geraniales. Dis sert. Bot. 11. p. 163.

- Perveen A. and Kaiser, M. 1999. Pollen Flora of Pakistan. Univ. of Karachi. pp. 263-269.

- Shehata, A. A. 2008. Pollen Morphology of Egyptian Geraniaceae: An Ass essment of Taxonomic value. Univ. of Alexandria, Egypt.pp. 67-76.

- Stafford, P. J. and Blakhmore, S. 1991. The Northwest European Pollen Flora. Geraniaceae Rev. Palaeon. Plynol. 69. pp. 49-78.

- Stafford, P. J. and Gibby, M. 1992. Pollen morphology of the genus Pelargonium (Geraniaceae). Rev. Paleobot. Palynol. 71. pp. 79-109.

- Verhoeven, R. L. and Venter, H. J. 1987. Pollen morphology of Erodium in South Africa. pp. 279-283.

- Walker, J. W. and Doyle, J. A. 1976. The basis of Angiosperm phylogen Palynology. Ann. Mo. Bot. Gard. 62. pp. 666-723. 